\title{
Bile salt deconjugation and cholesterol removal from media by Lactobacillus strains used as probiotics in chickens
}

\begin{abstract}
BACKGROUND: Bile salt deconjugation by Lactobacillus strains is often closely linked to bile tolerance and survival of the strains in the gut and lowering of cholesterol in the host. The present study investigated the deconjugation of bile salts and removal of cholesterol by 12 Lactobacillus strains in vitro. The 12 strains were previously isolated from the gastrointestinal tract of chickens. RESULTS: The 12 Lactobacillus strains could deconjugate sodium glycocholate (GCA, 16.87-100\%) and sodium taurocholate (TCA, 1.69-57.43\%) bile salts to varying degrees, with all strains except L. salivarius I 24 having a higher affinity for GCA. The 12 Lactobacillus strains also showed significant $(\mathrm{P}<0.05)$ differences in their ability to remove cholesterol from the growth medium $(26.74-85.41 \%)$. Significant $(\mathrm{P}<0.05)$ correlations were observed between cholesterol removal and deconjugation of TCA $(r=0.83)$ among the $\mathrm{L}$. reuteri strains $(\mathrm{C} 1, \mathrm{C} 10$ and $\mathrm{C} 16)$ and between cholesterol removal and deconjugation of TCA $(r=0.38)$ and GCA $(r=0.70)$ among the L. brevis strains (I 12, I 23, I 25, I 211 and I 218). In contrast, although L. gallinarum I 16 and I 26 and L. panis C 17 showed high deconjugating activity, there was no correlation between cholesterol removal and deconjugation of bile salts in these strains. CONCLUSION: The results showed that the 12 Lactobacillus strains were able to deconjugate bile salts and remove cholesterol in vitro, but not all strains with high deconjugating activity removed cholesterol effectively.
\end{abstract}

Keyword: Lactobacillus; Probiotics; Bile salts; BSH; Deconjugation; Cholesterol 